\title{
Negative association between previous allergy and intradermal tests for rocuronium and cisatracurium: what about additional tests?
}

\author{
Nicholas G. Kounis ${ }^{1}$, Ioanna Koniari ${ }^{2}$, Emmanouil Chourdakis ${ }^{3}$, \\ Periklis Davlouros ${ }^{1}$, and George Hahalis ${ }^{1}$ \\ ${ }^{1}$ Department of Cardiology, University of Patras Medical School, Patras, Greece, ${ }^{2}$ Department of Electrophysiology, \\ Queen Elizabeth University Hospital, Birmingham, England, ${ }^{3}$ Krankenhaus der Barmherzigen Brüder Trier, Trier, \\ Germany
}

In a retrospective analysis published in the Korean Journal of Anesthesiology [1] concerning the relationship between intradermal tests for neuromuscular blocking agents in patients with history of allergy to various anesthetic agents, no association between allergy history and positive skin test was found.

This report raises the following important issues with respect to anaphylaxis during anesthesia, rocuronium-sugammadex complex, additive anaphylactic effect of anesthetic agents, and additional diagnostic tests in anesthesia:

1. Anaphylaxis during anesthesia constitutes a severe adverse event, rendering its identification and early treatment imperative. and partially explains its causality, pathophysiology, and mortality. The incidence of hypersensitivity reactions during anesthesia varies from $1: 3,180$ to $1: 10,000$ based on several prospective studies; however, this incidence might be underestimated [2]. Multiple causative factors have been implicated, including drugs, liquids, metal devices, materials, and procedures

Corresponding author: Nicholas G. Kounis, M.D., Ph.D., FESC

Department of Cardiology, University of Patras Medical School, Rion, Patras 26504, Achaia, Greece

Tel: 302610279579 , Fax: 302610279579

Email: ngkounis@otenet.gr

ORCID: https://orcid.org/0000-0002-9751-6710

Received: 3 June 2018.

Revised: 13 June 2018.

Accepted: 14 June 2018.

Korean J Anesthesiol 2018 August 71(4): 328-329

https://doi.org/10.4097/kja.d.18.00150 during anesthesia. The incidence of perioperative reactions in Spain was $1: 381$, involving mild skin reactions (48\%) and cases of anaphylaxis (52\%). On rare occasions, skin rash might be absent, as seen with drug reactions in eosinophilia and Kounis syndrome.

2. Sugammadex induces selective reversal of aminosteroidal non-depolarizing neuromuscular blockers acting as muscle relaxants, such as rocuronium and vecuronium. Sugammadex is a modified gamma cyclodextrin with eight carboxyl thio ether groups at the sixth carbon positions, creating a cavity that can encapsulate the rocuronium molecule and further produce the rocuronium-sugammadex complex. The rocuronium-sugammadex complex can induce anaphylactic reactions, and is also suggested to induce Kounis syndrome [3]. Notably, during anesthesia, patients may be exposed to various agents such as propofol, remifentanil, rocuronium, fentanyl, and sugammadex, all able to induce immunological changes.

3. In a recent report [4], a 46-year-old male patient developed tachycardia with ST elevation in the inferior leads and shock without cutaneous manifestations following propofol, remifentanil, rocuronium, fentanyl, and sugammadex administration during anesthesia for laparoscopic surgery. Two years later, a similar anaphylactic reaction occurred, this time accompanied by generalized erythema, following perioperative administration of rocuronium and sugammadex. Serum histamine and tryptase levels were increased, whereas skin prick tests were negative for rocuronium and sugammadex but positive for histamine and rocuronium-sugammadex complex. The authors wondered how rocuronium-sugammadex complex formation could induce im-

(c) This is an open-access article distributed under the terms of the Creative Commons Attribution Non-Commercial License (http://creativecommons.org/ licenses/by-nc/4.0/), which permits unrestricted non-commercial use, distribution, and reproduction in any medium, provided the original work is properly cited. 
munological changes and allergic reactions. In another report [5] of anaphylactic shock induced by the rocuronium-sugammadex complex after cesarean section, intradermal skin tests were negative for sugammadex and positive for rocuronium. A follow-up skin prick test with pre-mixed rocuronium-sugammadex complex revealed a strong positive reaction, but a test with only rocuronium was negative.

4. It is well known that simultaneous exposure to several drugs, acting as antigens, can produce more extensive symptoms than mono-sensitization. Furthermore, immunoglobulin E (IgE) antibodies with different specificities can have an additive effect, and even small amounts of corresponding antigens can further trigger mediator release in cases of exposure.

5. Determination of the appropriate examinations to elucidate the pathogenesis and etiology of anaphylaxis in order to establish risk-reduction strategies seems to be of paramount importance. We suggest, therefore, that apart from intradermal skin tests, serum histamine, serum tryptase, eosinophils, and total IgEs, the following tests would be of additional value [6]:

a. Serum-specific IgE measurements for the suspected drug;

b. Radioallergosorbent testing, enzyme-linked immunosorbent assay or fluoroenzyme immunoassay.

c. Drug provocation test, which is a controlled challenge with the drug suspected of causing the hypersensitivity reaction; and

d. Basophil activation test, which is a cytometry method of measurement of drug-induced activation of basophil markers CD63 or CD203c.

\section{ORCID}

Nicholas G. Kounis, https://orcid.org/0000-0002-9751-6710

Ioanna Koniari, https://orcid.org/0000-0002-1033-5299

Emmanouil Chourdakis, https://orcid.org/0000-0001-5859-7944

Periklis Davlouros, https://orcid.org/0000-0002-1439-1992

George Hahalis, https://orcid.org/0000-0002-1483-1353

\section{References}

1. Kim YY, Kim IT, Shin SI, Yim SM. Intradermal skin tests for rocuronium and cisatracurium in patients with a history of allergy: a retrospective study. Korean J Anesthesiol 2018; 71: 296-9.

2. Malinovsky JM, Decagny S, Wessel F, Guilloux L, Mertes PM. Systematic follow-up increases incidence of anaphylaxis during adverse reactions in anesthetized patients. Acta Anaesthesiol Scand 2008; 52: 175-81.

3. Yang HS, Kim HJ, Koh W. Effects of sugammadex on the coronary circulation: direct effects on coronary vessels or hypersensitivity (Kounis syndrome)? Korean J Anesthesiol 2017; 70: 363-4.

4. Okuno A, Matsuki Y, Tabata M, Shigemi K. A suspected case of coronary vasospasm induced by anaphylactic shock caused by rocuroniumsugammadex complex. J Clin Anesth 2018; 48: 7.

5. Yamaoka M, Deguchi M, Ninomiya K, Kurasako T, Matsumoto M. A suspected case of rocuronium-sugammadex complex-induced anaphylactic shock after cesarean section. J Anesth 2017; 31: 148-51.

6. Rive CM, Bourke J, Phillips EJ. Testing for drug hypersensitivity syndromes. Clin Biochem Rev 2013; 34: 15-38. 